\title{
Les passions comme causes dans la Rhétorique d'Aristote: mobiles de l'action et instruments de la persuasion ${ }^{*}$
}

Dans la Rhétorique, Aristote attribue aux passions une causalité complexe: dans I, 10-13, elles jouent le rôle de mobiles de l'action injuste et dans II, 1-12, elles figurent comme des instruments techniques de la persuasion: on les suscite chez les auditeurs afin d'influencer leur jugement. Mon but sera de répondre aux questions suivantes: (a) quelles formes de causalité émergent de ces deux différentes évaluations des passions ? (b) L'analyse des passions et notamment la liste des passions de Rhet. II, nous permet-elle de mieux comprendre la nature du traité aristotélicien?

La Rhétorique est un texte capital pour tenter de reconstituer ce puzzle difficile et déroutant qu' est la théorie aristotélicienne des passions.

En premier lieu, parce que les passions y jouent un rôle fondamental. En effet, dans la perspective de la réhabilitation de l'élément émotif dans la persuasion qu'Aristote opère, par contraste avec la vision platonicienne, les passions ne sont plus des facteurs négatifs de la nature humaine dont la manipulation est le propre d'une rhétorique trompeuse et abusive. Elles apparaissent au contraire comme le "facteur humain" incontournable qui détermine tous les rapports sociaux dans la polis et avec lequel tout orateur doit se confronter.

Michel Meyer considérait le facteur émotif comme l'équivalent de la moderne "conscience de soi". Il écrivait à ce sujet:

Pour les Grecs, les passions sont avant tout politiques, au sens large du terme: elles marquent la vie de la Cité, c'est-à-dire les rapports qu'entretiennent les hommes libres entre eux. (...) Sièges de l'individu avant la lettre, les passions sont les signes des

\footnotetext{
${ }^{*}$ Ce texte résume les séances du séminaire que j'ai tenu à São Paulo (USP) en août 2009 dans le cadre du programme de coopération scientifique France - Brésil - "Aristote : causes et actions » (programme CNRS / FAPESP 22137). Je remercie Marco Zingano, Carlo Natali et tous les autres participants pour leurs remarques et suggestions ainsi que mon amie Jocelyne Wilhelm pour sa relecture toujours précieuse.
} 
Journal of Ancient Philosophy Vol. IV 2010 Issue 1

différences, de la diversité humaine, avec lesquelles il faut compter pour pouvoir vivre ensemble. Elles sont aussi ce par quoi se manifestent les oppositions, elles les traduisent, donc les communiquent. On pense à la colère, par exemple. En somme, la passion est ce qu'on appellera, quelques siècles après, la conscience (de soi), puisqu'elle exprime une réaction à autrui, une image de soi comme étant vue par l'autre, une réflexivité, un spectacle, une conscience observante ${ }^{1}$.

L'importance des passions dans la Rhétorique se manifeste notamment dans le domaine de la rhétorique judiciaire où elles jouent à la fois le rôle causal de mobiles des actions délictueuses (I, 10-13) et le rôle instrumental d'outils persuasifs pour influencer les juges au tribunal pendant un procès (II, 1-12).

En second lieu, parce que la Rhétorique est aussi notre source principale sur la théorie aristotélicienne des passions. En effet, Aristote développe justement dans la plus grande partie du livre II, une longue analyse des passions dans le but explicite de mettre en lumière tout ce qui est persuasif dans les états émotifs et de donner ainsi à l'orateur des outils pour agir efficacement sur le jugement de l'auditoire. Aristote produit ici celle qui a été appelée la "rhétorique des passions" ${ }^{2}$. Mais en réalité, cette analyse des passions de la Rhétorique semble dépasser largement son but purement technique et déployer un tableau extrêmement riche des états psychologiques et de leurs conséquences morales dans les rapports humains. Elle offre de fait l'examen le plus complet que nous pouvons trouver dans le corpus aristotélicien de complexes mécanismes d'action et de réaction des émotions fondamentales. La critique s'est beaucoup interrogée sur le rôle et le statut de cette "rhétorique des passions" dans le corpus aristotélicien. Elle a été vue aussi bien comme la prémisse ${ }^{3}$, que comme la

\footnotetext{
${ }^{1}$ M. Meyer, Le philosophe et les passions. Esquisse d'une histoire de la nature humaine, Paris, 1991, p. 14.

${ }^{2}$ Cf. P. Aubenque, "Logos et pathos. Pour une définition dialectique des passions (De anima, I, 1 et Rhétorique, II)" , dans Corps et âme. Sur le De anima d'Aristote, G. Romeyer- Dherbey et C. Viano éd., Paris, 1996, p. 46: «Ce que propose le livre II n’est donc pas une psychologie des passions entendue comme réquisit scientifique, mais bien une rhétorique des passions, supposée seule adéquate à fournir en arguments un discours rhétorique destiné à agir sur l'affectivité». $C f$. le titre du livre de $\mathrm{M}$. Meyer, Aristote ou la Rhétorique des passions, Paris, 1989 et le paragraphe intitulé "La rhétorique des passions" dans la préface de la trad. de Ch.-E. Ruelle de la Rhétorique d'Aristote, Paris, 1991, p. 5153: ici l'expression indique l'habileté à susciter les passions par le discours.

${ }^{3}$ Cf. J. Cooper, “On Aristotelian Theory of Emotions”, dans E. Oksenberg Rorty (ed.), Essays on Aristotle's Rhetoric, Berkeley-Los Angeles-London 1996, p. 238-57; P. Aubenque, "Logos et pathos", art. cit. et "Sur la définition aristotélicienne de la colère", Revue philosophique (147, 1957), p. 300317.
} 
Journal of Ancient Philosophy Vol. IV 2010 Issue 1

conséquence ${ }^{4}$ de cette enquête "scientifique" et exhaustive sur les passions, censée rendre compte à la fois de la "forme" et de la "matière" de celles-ci, dont Aristote esquisse le modèle dans le De anima ${ }^{5}$ mais qu'en réalité il ne développera jamais, du moins dans le corpus qui nous est parvenu ${ }^{6}$. La définition de la nature de cette enquête sur les passions dans la Rhétorique rejoint ainsi la question de la compréhension de la nature du traité rhétorique luimême: la Rhétorique d'Aristote est-elle de fait un manuel pratique destiné à former les orateurs ou bien une réflexion théorique, qui comprend et dépasse son but "technique" et déclaré de la persuasion?

Aristote lui-même déclare le caractère "théorique" de cette discipline tout au début du traité: "Nous définissons la rhétorique comme la capacité de reconnaître dans chaque cas ce qui est

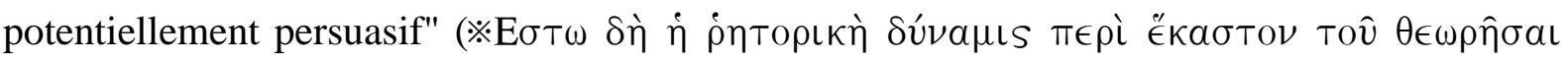

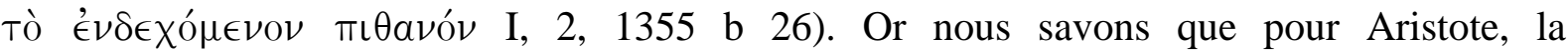
connaissance théorique signifie en premier lieu connaître les causes.

C'est donc dans la perspective de leur causalité complexe que je me propose d' aborder les passions dans la Rhétorique, et précisément dans I, 10-13, où Aristote, au sujet du genre judiciaire, analyse les passions en tant que mobiles de l'action et dans II, 1-12, où il présente

\footnotetext{
${ }^{4}$ Cf. par ex. G. Striker, "Emotions in Context: Aristotle's Treatment of the Passions in the Rhetoric and His Moral Psychology”, dans E. Oksenberg Rorty (ed.), Aristotle's Rhetoric, p. 286-302.

${ }^{5}$ De anima, I, 1, 403 a 24 suiv.: "Dans ces conditions, il est évident que les affections sont des formes engagées dans la matière. Aussi les définitions doivent-elles être formulées en conséquence, par exemple: la colère est le mouvement d'un corps donné, d'une partie ou d'une faculté de ce corps, produit par telle cause en vue de telle fin. Voilà pourquoi il appartient donc au physicien de traiter l'âme, soit de toute espèce d'âme, soit de l'âme telle que nous la décrivons. "

${ }^{6}$ Dans le catalogue des ouvrages d'Aristote de Diogène Laërce (V, 22-27), mention est faite d'un peri pathôn orgês mais il s'agit probablement d'un ouvrage péripatéticien postérieur à Aristote; $c f$. $\mathrm{P}$. Moraux, Les listes anciennes des ouvrages d'Aristote, Louvain, 1951, p. 74 suiv. R. Laurenti, Aristotele, I frammenti dei dialoghi, Napoli, 1987, vol. I, p. 305 suiv. et J. Fillion-Lahille, "La colère chez Aristote", Revue des Etudes anciennes 72 (1970), p. 46-79, supposent en revanche qu'il s'agit d'un traité d'Aristote. Selon J. Fillion-Lahille le titre originaire aurait été Peri pathôn et orgês une glose, expliquée par le fait qu'Aristote y aurait développé largement la colère en exprimant très sensiblement son indulgence à son égard. Cela expliquerait aussi la polémique antipéripatécienne suivante sur ce point. Aspasius, In Eth. Nic. 44, 19 remarque que l'on ne trouve aucune définition d'émotion chez aucun membre de l'école péripatéticienne. Selon W.W. Fortenbaugh, "Aristotle's Rhetoric on Emotions", Archiv für die Geschichte der Philosophie 52 (1970), p. 61, Aristote aurait traité de manière systématique les définitions des émotions dans un ouvrage perdu comme les Divisions et ensuite inséré ces définitions dans la Rhétorique. Une trace de cela serait le critère de division (diairein) qu'il applique à l'analyse des passions de Rhet. II: dispositions de l'âme, contre qui et sur quels sujets.
} 
les passions en tant qu'instruments techniques de la persuasion, à savoir comme causes du jugement qu'il faut susciter chez les juges.

Mon but sera de répondre aux questions suivantes:

(a) Quelles formes de causalité sont attribuées aux passions par Aristote? Comment s'articulent-elles entre elles? Qu'y a-t-il en commun entre ces deux évaluations des passions? Peut-on ramener leurs actions à un modèle unique de causalité?

(b) L'analyse des passions et notamment la liste des passions de Rhet. II, nous permet-elle de mieux comprendre la nature du traité aristotélicien?

I. Les passions comme causes de l'action: la rhétorique judiciaire (I, 10-15)

\section{I.1. L'acte injuste et ses causes}

Dans le livre I de la Rhétorique, Aristote distingue les trois genres oratoires: délibératif, judiciaire et épidictique. Leurs facteurs distinctifs sont: les auditeurs (membres de l'assemblée, jurés d'un tribunal populaire, spectateurs d'un discours de cérémonie), le temps auquel ils se référent (futur, passé, présent) et la fin visée (utile ou nuisible, juste ou injuste, beau ou laid).

De fait, l'analyse du genre judiciaire est aussi la plus étendue et occupe les ch. 10-15 du premier livre. Dans ce contexte, Aristote définit une série de notions fondamentales du droit pénal et de la réflexion juridique, que l'on retrouve aussi de manière plus détaillée dans les Ethiques et qui sont à la base des juridictions successives jusqu'à nos jours, comme la notion d'acte injuste (adikein) et de ses causes, de volontaire/involontaire (hekon/akousion), de choix délibéré (proairesis), de responsabilité (di'autous), de culpabilité (aitios), d'équité (epieikeia). Les passions rentrent ici dans les causes de l'action. Comme nous le verrons, Aristote exprime cette causalité en distinguant deux aspects fondamentaux de l'action: la cause finale et la cause efficiente.

Or, les causes de l'action rentrent dans les prémisses qui servent à construire les arguments (enthymèmes, syllogismes) du discours judiciare, à savoir l'accusation et la défense:

En ce qui concerne l'accusation et la défense, on peut dire dans la foulée le nombre et la nature des prémisses d'où doivent être tirés les syllogismes. Trois points sont à considérer. Premièrement, quels sont, et en quel nombre, les mobiles pour lesquels les gens commettent des injustices; deuxièmement quelles sont alors les dispositions de ces 
Journal of Ancient Philosophy Vol. IV 2010 Issue 1

gens; troisièmement de quels types sont leurs victimes et quelles sont les dispositions de celles-ci ${ }^{7}$.

L'analyse porte sur la qualification de trois éléments: les causes de l'action, la disposition de l'agent, la disposition et la qualité du patient. Quant aux causes, on remarquera qu'il s'agit

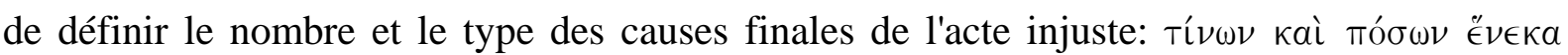

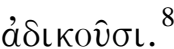

On remarquera que l'évaluation d'un délit n'est pas unilatérale et figée, mais tient compte aussi de l'interaction des circonstances, des caractères et des états psychiques de l'agent et du patient. Ce schéma d'analyse tripartite nous le retrouverons dans le livre II, 1, 1378 a 22, appliqué aux passions en tant qu'instruments de persuasion: il faudra saisir la disposition d'âme (pôs) de celui qui éprouve l'émotion, à l'égard de quelles personnes (tisin) et à propos de quels sujets (tisin).

Aristote définit ensuite l'acte injuste dans un passage très dense où il précise chaque terme de la définition:

Nous définissons l'acte injuste comme le fait de nuire volontairement en violation de la loi. La loi est soit particulière, soit commune. Par loi particulière, j'entends la loi écrite qui régit chaque cité; par lois communes, celles qui, sans être écrites, semblent être reconnues par le consentement universel. Les actions volontaires sont celles que l'on accomplit en sachant ce qu'on fait et sans y être forcé. Toutes les actions volontaires ne proviennent pas d'un choix délibéré, mais tout ce qu'on fait selon un choix délibéré, on le fait en connaissance de cause, car personne n'ignore ce qu'il choisit délibérément ${ }^{9}$.

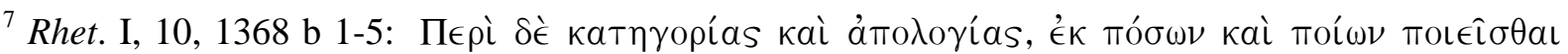

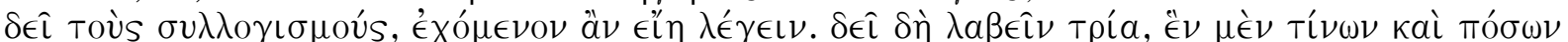

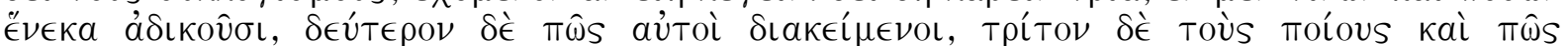
ÉXovtas. Pour le texte de la Rhétorique, je vais suivre, dans la plupart des cas, la récente traduction de P. Chiron, Aristote, Rhétorique, Paris, 2007, en lui apportant quelques modifications.

8 D. Davidson , "L' action", dans J. Benoist et alii, Quelle philosophie pour le XXIe siècle? Paris, 2001, p. 300-331, ici p. 308, suit complètement Aristote sur ce point :" Dans le cas de l'action humaine, ce que nous recherchons, c'est la compréhension: nous voulons savoir pourquoi quelqu'un agit en fonction des ses croyances ou de ses désirs ou d'autres attitudes"

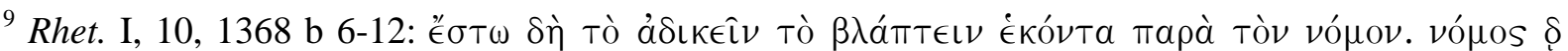

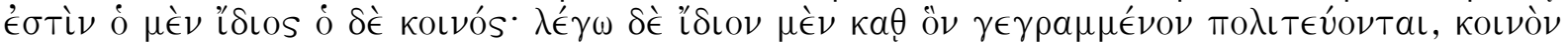

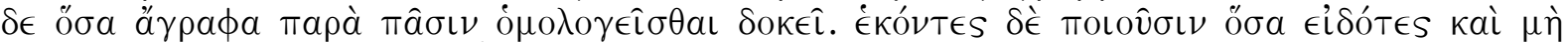

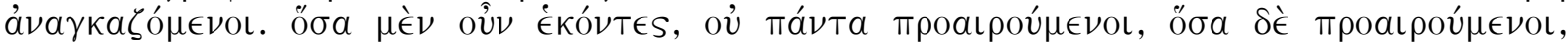

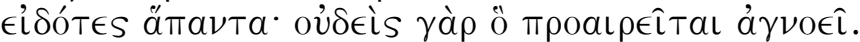


On remarquera tout d'abord que la définition de l'acte injuste est précédée par estô dê, "soit donc": cette formule est fréquente dans le traité pour définir les notions "techniques" de l'art rhétorique. En effet, la première occurrence se trouve en 1355 b 26 pour définir la rhétorique elle-même comme la capacité de reconnaître ce qui est persuasif. Nous allons la retrouver dans le livre II pour introduire les définitions des passions ${ }^{10}$. Aristote distingue ensuite les causes générales des causes particulières de l'acte injuste.

Les causes générales sont le vice et l' akrasia : "Les raisons pour lesquelles on choisit délibérément de nuire à autrui et de faire le mal en violant la loi, sont le vice et l'incapacité à

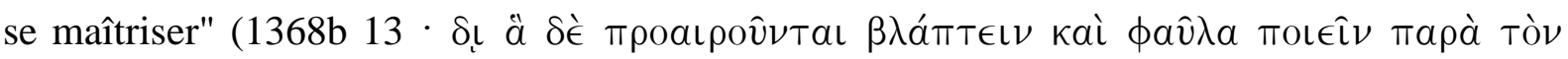

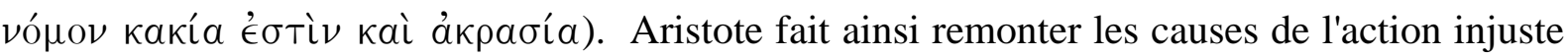
aux deux mauvaises dispositions (hexeis) à l'égard des passions: l'une acquise et systématique, le vice, et l'autre transitoire et susceptible d'être récupérée, l'incapacité à se maîtriser. De fait le vice est rare et l'akrasia est la disposition psychique la plus répandue parmi les hommes. Aristote précise ensuite (b15-24) que celui qui a différents vices, sera injuste relativement à chacun. Il présente une liste de caractères qu'il relie parfois au pathos correspondant, par exemple, l' emporté par rapport à la colère (di'orgên, b 20). Pour l'explication de ces sujets, il renvoie à ce qu'il a dit sur la vertu et à ce qu'il dira sur les passions (b 25). Grimaldi observe justement ${ }^{11}$ que la connaissance des vertus est utile dans ce contexte parce qu'elle permet de reconnaître leurs déviations, de même que celle des émotions permet de distinguer les bonnes des mauvaises. On peut supposer qu'Aristote ici renvoie à l'analyse des passions du livre II.

Quant aux causes particulières:

Il nous reste à dire pourquoi (= à quelles fins) les gens commettent l'injustice, dans quelles dispositions ils sont alors et qui sont leurs victimes. Distinguons d'abord quelles sont les impulsions et les répulsions qui animent les hommes quand ils entreprennent de commettre l'injustice. C'est clair que l'accusateur doit se préoccuper de savoir parmi les mobiles qui poussent les hommes à commettre l'injustice envers leur prochain, combien et de quelle nature sont celles qui sont imputables à son adversaire, et le

\footnotetext{
${ }^{10}$ Les interprètes sont partagés sur la valeur à donner à cette formule: les uns la considèrent comme une marque du caractère populaire des définitions qu'elle précède, les autres, au contraire, comme une marque du caractère scientifique de ces définitions. Pour le sens de cette formule, voir plus loin, n. 33 .

${ }^{11}$ W.M.A. Grimaldi, Aristotle, Rhetoric I, A commentary, New York, 1980, p. 230.
} 
Journal of Ancient Philosophy Vol. IV 2010 Issue 1

défenseur doit se préoccuper de savoir de quelle nature et en quel nombre sont, parmi ces choses, celles qu'on ne peut lui imputer ${ }^{12}$.

Ce passage montre combien est importante l'évaluation des mobiles (les objets du désir, à savoir les causes finales) d'un acte injuste pour l'accusation et pour la défense dans un procès. Ensuite Aristote précise la cause efficiente et définit la responsabilité de l'acte: "Or dans tous les cas, l'agent quel qu'il soit ou bien n'est pas la cause ou bien est la cause de l'action" (1368b

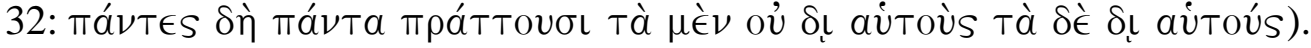

Aristote (1368 b 32) distingue sept causes de l'acte injuste. Les unes sont extérieures à l'agent, et dans ce cas, ce n'est pas lui la cause: il s'agit de la fortune (tuchê) et de la nécessité (ananchê), qui est tantôt constriction (bia), tantôt naturelle (phusei). Les autres sont intérieures à l'agent et dont l' agent est responsable (aitios) : il s'agit de l' habitude (ethos), du désir (orexis) rationnel, à savoir le raisonnement (logismos), et des deux formes du désir irrationnel, à savoir, l'emportement (thumos) et le désir sensuel (epithumia).

De fait, en 1368 b 37-a 4, Aristote réduit les causes efficientes de l'action responsable à deux facteurs:

Tous les actes dont l'agent est la cause, c'est-à-dire les actes dont les hommes sont euxmêmes responsables, sont imputables les uns à l'habitude, les autres au désir - que ce soit un désir rationnel ou un désir irrationnel ${ }^{13}$.

En réalité, ethos et orexis ne se posent pas comme causes alternatives de l'action. Ici ethos semble être, dans le domaine de l'action responsable, le corrélatif de phusis, car il exprime une "seconde nature", une disposition stable de l'âme résultant de la répétition des mêmes actes à agir d'une certaine manière ${ }^{14}$. Mais, d'autre part, dans l'homme l'ethos n'est pas un comportement purement mécanique, dicté par la nature, comme chez les animaux, car il consiste en dernière instance en une attitude constante à l'égard des pathê. L'ethos est d'ailleurs l'habitude sur la quelle se forment l'hexis, la disposition stable et le caractère

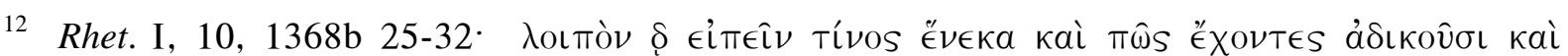

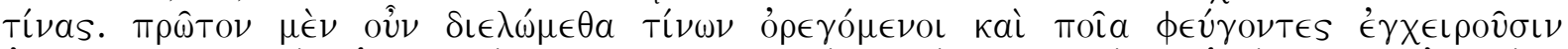

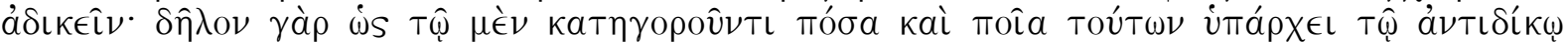

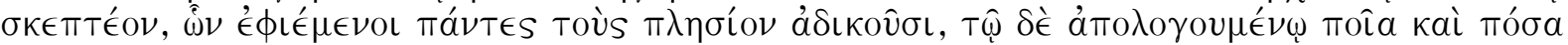

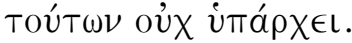

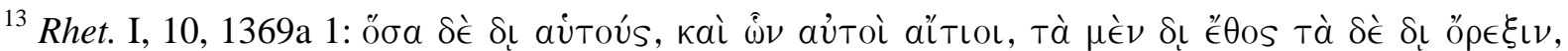

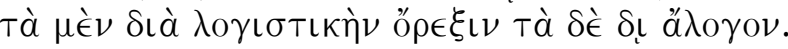

${ }^{14}$ Cf. Grimaldi, I, p. 246: habitual way of acting. 
Journal of Ancient Philosophy Vol. IV 2010 Issue 1

(êthos) ${ }^{15}$. A ce propos, Aristote en 1369a 15, explique que les actions des justes et des injustes qui sont dites dériver de leurs dispositions (hexeis), sont reconduisibles, en fin de comptes, au logismos et au pathos.

En effet, en 1369a 7 suiv., Aristote opère une réduction ultérieure des causes de l'action au calcul (logismos) et au pathos (epithumia et orgê), ce qui revient à identifier la cause efficiente de l'action à la catégorie unique de l'orexis, du désir, rationnel et irrationnel.

A partir de 1369 a 32, Aristote décrit en détail les sept causes de l'action : ici, on voit bien qu'il fait la distinction entre cause motrice et cause finale dans une action. Par exemple, à propos de la tuchê, nous lisons: "sont dus à la chance tous les faits tels que la cause est indéterminée (aitia aoristos) et qui ne se produisent pas en vue d'une fin (kai mê heneka)" (1369a 32).

En ce qui concerne les actions responsables, Aristote analyse la finalité des différentes formes de désir: le calcul (dia logismon, 1369b 7) vise des actes utiles pour obtenir des biens tant à titre de fins que de moyens pour en obtenir d'autres ; le désir impulsif (1369 b 11: dia thumon kai orgên) concerne les actes de vengeance (timôrêtika) dont la fin coïncide avec la satisfaction de l'agent (hina plerôthê). Quant aux actes accomplis par désir irrationnel et sensuel (1369a 15: dia epithumian), la fin coïncide avec "tout ce qui paraît agréable" (hêdu).

Aristote en conclut que tous les actes dont on est la cause sont ou apparaissent bons (agatha) ou agréables (hêdea). Aristote aboutit ici, dans le domaine des actes injustes, à une cause finale unifiée, qui est le plaisir, de même que, dans l'analyse des actions responsables, il avait abouti à une causalité efficiente unifiée qui est le désir. De fait, l'argumentation d'Aristote pour parvenir à cette réduction des mobiles de l'action injuste au plaisir est assez elliptique: s'il écarte explicitement l'utile en le réservant au genre délibératif, la réduction ici du bon à l'agréable en revanche n'est pas très explicite. On peut comprendre cela à la lumière de l'analyse du bien et de l'utile de I, 6, 1362 b 5 suiv. au sujet du genre délibératif et où il considère le plaisir comme un bien car "tous les êtres le désirent naturellement" (ephietai ...tê phusei) et dit que les choses belles et agréables sont aussi plaisantes (hêdea). Vu que le bon et l'utile font l'objet propre du genre délibératif, l'agréable semble faire l'objet propre du judiciaire et apparaît ainsi comme le mobile fondamental des actes injustes. Comme nous le verrons, dans la plupart des définitions du livre II, les passions sont présentées justement comme des formes de douleur dont la cessation engendre le plaisir.

\section{I.2. Le plaisir (et la douleur)}

\footnotetext{
${ }^{15}$ Pour les rapports de dérivation entre ethos, êthos, hexis, voir Grimaldi, I, p. 233, ad a 8.
} 
Journal of Ancient Philosophy Vol. IV 2010 Issue 1

La suite (I, 11) est consacrée justement à l'analyse du plaisir (hêdonê). Cette analyse est précédée par une considération méthodologique qui a été considérée comme emblématique de tout le traité : "Parlons maintenant de l'agréable. On admettra comme suffisantes des définitions qui, sur chaque point, ne sont "ni obscures ni exactes" (mête asapheis, mête akribeis, 1369 b 31-32). Aristote définit ainsi le plaisir: "Supposons que le plaisir est un mouvement de l'âme et un retour total et sensible à l' état naturel, et que la peine est l'inverse"16.

Cette définition du plaisir soulève tout de suite un problème car il s'agit d'une définition du plaisir qu'Aristote critique dans Eth. Nic. X, 2, 1173 a 28 suiv. et VII, 12, 1152 b 13-15 (cf. aussi Top. IV, 1, 121 a 27 suiv.). En effet, dans Eth. Nic. X, 4, 1174 b 23, Aristote nie justement que le plaisir soit un mouvement (energeia) et le considère plutôt comme un perfectionnement de l'acte (epigignomenon ti telos). Chaque plaisir est étroitement lié à l'acte qu'il amène à l'accomplissement (5, 1175 a 29: sunôkeiôsthai). Les plaisirs suivent (hepontai) les activités et les perfectionnent (1176 a 25). De fait, la définition de plaisir comme katastasis, on la retrouve chez Platon, Philèbe, 42 c-d.

On remarquera que cette définition est précédée par l'expression hupokeisthô : "supposons" ne semble pas indiquer la formulation d'une véritable définition propre à la discipline rhétorique (cf. diorisamenoi, estô dê, à propos de l'acte injuste) mais plutôt la formulation d'une hypothèse de travail, qui est pourtant valable et utile pour satisfaire les auditeurs, ce qui est cohérent avec la déclaration méthodologique ci-dessus ${ }^{17}$. Parmi les interprètes, Grimaldi montre que les différences avec l' Eth. Nic. ne sont pas si grandes, surtout si l'on prend en compte le développement de VII, 11-14. En particulier, en VII, 13, 1153 a 2, Aristote remarque que les hommes "n' éprouvent pas plaisir pour les mêmes choses quand il se trouvent dans le processus de réintégration de leur propre nature et quand telle nature est déjà

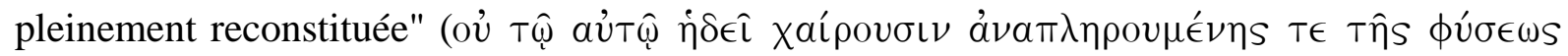

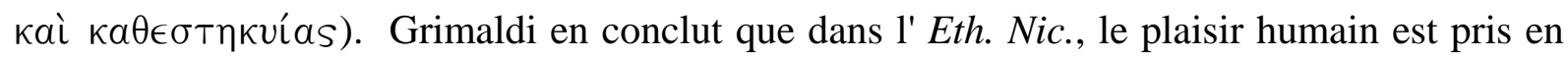
considération par rapport aux activités de la nature humaine et que dans la Rhétorique le

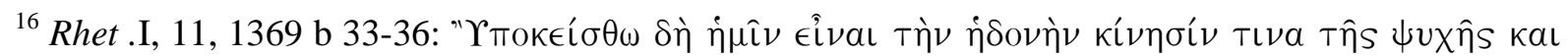

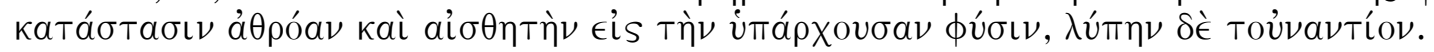

${ }^{17}$ Grimaldi, I, p. 243.
} 
plaisir est évalué par rapport à la nature humaine tout court ${ }^{18}$. Cette interprétation me semble plausible et on pourrait identifier la "nature humaine" en question à son niveau le plus "basique", celui de l'intégrité psychique, mais aussi physique. Il s'agit donc d'une définition du plaisir qui n'est pas en contradiction avec le développement de l'Ethique à Nicomaque, mais qui en met en lumière un aspect qui intéresse la rhétorique.

En effet, on remarquera qu'aussi bien en ce qui concerne les désirs purement irrationnels et sensuels (soif, faim et sexe), que les désirs rationnels, comme le désir d'apprendre, les actions agréables présentent le caractère commun d'un retour à l'état naturel ou bien de la réalisation de la plénitude de sa nature ou de ce qui est conforme à sa propre nature.

En particulier, Aristote analyse ici un certain nombre de désirs non physiques qui s'accompagnent du plaisir de l'imagination. C'est le cas emblématique de la colère, pathos par sa nature douloureuse, suscité par le manque de considération, qui s'accompagne pourtant du plaisir dû à l'espoir de la vengeance. En effet, la vengeance consiste à rendre la pareille en rétablissant ainsi un état d'équilibre et d'intégrité psychique qu'un acte de mépris a altéré. P. Aubenque a très bien parlé de "réattestation de soi" ${ }^{19}$. On pourrait aussi désigner ce processus en termes psychologiques modernes de "reconstruction de soi".

Outre la colère, nous trouvons ici, parmi les plaisirs non physiques un certain nombre d' états psychologiques agressifs et compétitifs que l'on retrouvera à l'arrière plan des analyses spécifiques des passions sociales du livre II et qui illustrent bien cette conception du plaisir comme attestation de soi et en développent certains aspects.

Par exemple, en 1370 b 32 Aristote explique que la victoire est agréable (hêdu) non seulement pour les ambitieux mais aussi pour tout le monde «car il se produit une imagination de supériorité (phantasia huperochês) dont tous les hommes ont le désir plus ou moins fort». Le plaisir lié à l'espoir de la victoire explique à son tour le plaisir que l'on éprouve dans les activités compétitives telles que les jeux, la chasse, les batailles, les disputes, la lutte et l'éristique. Une autre source de plaisir est l'amour que chacun ressent pour soi-même (philautos) (1371 b 18), qui est antérieur à l'amitié et qui détermine toutes les formes d'affection pour les autres ${ }^{20}$. Enfin le plaisir de commander est associé à la sagesse pratique, et le plaisir de commander et de dominer son prochain est relié à l'ambition propre à tous les hommes (1371 b 26). En fait, philotimia (litt. «amour des honneurs») se traduit aussi bien par

\footnotetext{
${ }^{18}$ Grimaldi, I, p. 246.

${ }^{19} \mathrm{P}$. Aubenque, «Sur la définition aristotélicienne de la colère», p. 310.

${ }^{20}$ Eth. Nic. IX 8, 1168 b 9: «C'est en partant de cette relation de soi-même à soi-même que tous les sentiments qui constituent l'amitié se sont par la suite étendus aux autres hommes".
} 
Journal of Ancient Philosophy Vol. IV 2010 Issue 1

ambition que par rivalité. Elle naît entre les semblables et peut interagir de manière étonnante avec l'affection (philia).

Dans ce passage de la Rhétorique, Aristote montre qu'à l'origine des états émotionnels compétitifs comme les plaisirs du jeu et de la lutte, dans le fait de commander et de dominer les autres, il y a un désir d' auto-affirmation, d'attestation de soi et de sa propre supériorité, qui est naturel, universellement partagé et qui apparaît de fait comme l'un des mobiles fondamentaux des actions des hommes dans la société. Ainsi la définition de plaisir comme attestation et "réintégration" de soi face à la communauté se prête bien à son utilisation dans le contexte de la Rhétorique, qui concerne justement une activité éminemment sociale et interactive de l'homme : la persuasion de ses semblables.

\section{I.3. Qualification des délits}

Dans le chapitre 13, Aristote reprend la définition de l'acte injuste dans la perspective de la personne lésée: c'est souffrir des actes injustes accomplis volontairement par l'agent (13, 1373b 27)

L'action injuste est évaluée ainsi comme un dommage que la personne lésée subit (blaptêsthai, 1373b 30) contre sa volonté:

De sorte que, nécessairement, toutes les inculpations concernent soit la communauté soit l'individu, elles visent des actes commis soit dans l'ignorance et contre son gré, soit volontairement et en connaissance de cause, ces actes sont, dans ce dernier cas, le fruit soit d'un choix délibéré soit de la passion ${ }^{21}$.

La qualification (epigramma, 1374 a 1) d'un délit est justement déterminée par le choix délibéré:

Dans tous les cas de ce genre, il y a un débat sur la question de savoir si la personne est injuste et mauvaise ou non. Car la méchanceté et l'injustice résident dans le choix délibéré; et les termes de ce genre signifient le choix délibéré, par exemple les termes "outrage" et "vol"; car outrager n'est pas dans tous les cas frapper, mais frapper pour une

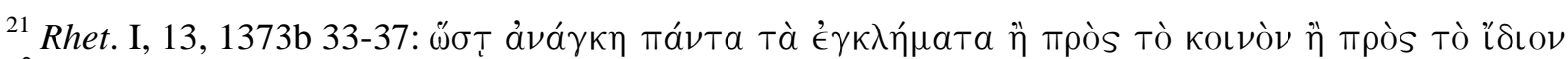

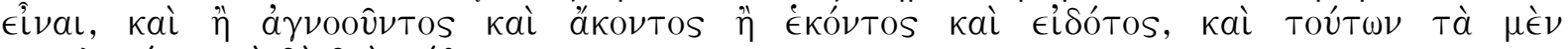

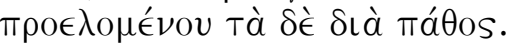


Journal of Ancient Philosophy Vol. IV 2010 Issue 1

fin déterminée, par exemple le déshonneur de celui que l'on frappe ou sa propre jouissance $^{22}$.

Aristote montre qu'entre action volontaire et choix délibéré, il n'y a pas de réciprocité: si toutes les actions qui proviennent d'un choix délibéré sont volontaires, cela ne signifie pas que toutes les actions volontaires proviennent d'un choix délibéré.

Aristote élabore de manière plus détaillée ces distinctions à l'intérieur de la catégorie de actes volontaires dans Eth. Nic. III et V, 10, où il analyse les notions de juste et de volontaire. Aristote définit ainsi le volontaire et explique ce que signifie "en connaissance de cause" (eidôs):

J'entends par volontaire, comme il a été dit précédemment, tout ce qui, parmi les choses

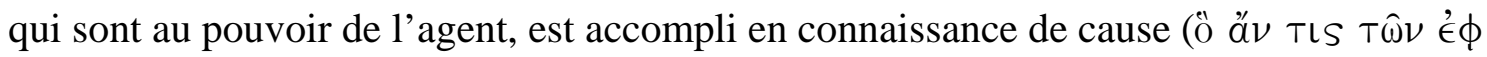

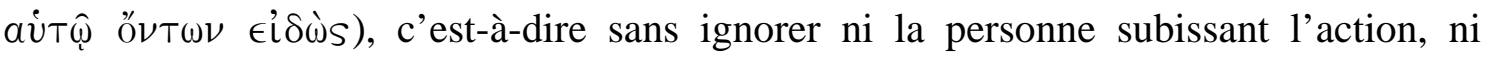
l'instrument employé, ni le but à atteindre (par exemple, l'agent doit connaître qui il frappe, avec quelle arme et en vue de quelle fin), chacune de ces déterminations excluant au surplus toute idée d'accident, ou de contrainte (V, 10, 1135 a 23-27).

En 1135 b 8 suiv., Aristote détaille les actes dommageables (blabai) à la lumière de cette distinction entre actes volontaires avec choix délibéré et sans choix délibéré. Il distingue ainsi: (a) actes involontaires (akousia) qui ont comme cause l' ignorance (le principe peut résider en nous ou venir du dehors), ce sont les torts (fautes, méprises); (b) actes volontaires (hekousia), ce sont les actes injustes. Mais (b.1) "Quand l'acte est fait en pleine connaissance, mais sans délibération préalable, c'est un acte injuste, par exemple tout ce qu'on fait par colère, ou par quelques autres de ces passions qui sont irrésistibles ou qui sont la conséquence de l'humaine nature (car en commettant ces torts et ces fautes les hommes agissent injustement, et leurs actions sont des actions injustes, bien qu'ils ne soient pas pour autant des êtres injustes ni pervers, le tort n’étant pas causé par méchanceté)" (1135 b 19-24). (b.2) "Mais quand l’acte procède d'un choix délibéré, c'est alors que l'homme est injuste et méchant" (adikos kai

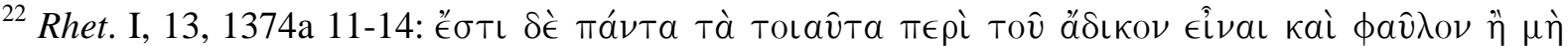

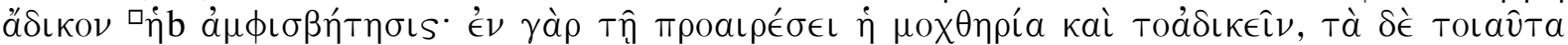

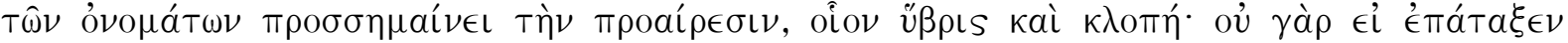

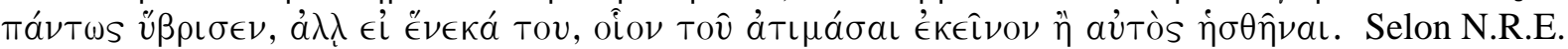
Fisher, Hybris. A Study in the Value of Honor and Shame in ancient Greece, Warminster, 1988, le focal meaning de la notion grecque de hubris - à la fois légal, politique et moral- est justement exprimé par la définition aristotélicienne de la Rhétorique d'insulte délibérée.
} 
mochthêros, b 25). D'où la distinction, dans le droit attique, entre un cas de coup et blessure avec préméditation (dikê traumatos ek pronoias) et sans préméditation. Dans le premier cas, la préméditation était identifiée à l'intention de tuer et l'action légale était donc de la compétence de l'Aréopage, qui traitait justement les actions de meurtre volontaire et prémédité.

On remarquera que dans cette description, l'individu qui commet une injustice sous l'emprise de la colère n'est pas lui-même injuste. Aristote accentue ultérieurement cet aspect de la colère de manière paradoxale:

De là vient que les actes accomplis par colère sont jugés à bon droit comme faits sans préméditation car ce n’est pas celui qui agit par colère qui est le véritable auteur du dommage, mais bien celui qui a provoqué sa colère. En outre, le débat ne porte pas sur la question de savoir s’il s'est produit ou non un fait dommageable, mais s’il a été causé justement (puisque c'est l'image d'une injustice qui déclenche la colère). ${ }^{23}$

Ce passage est assez étonnant puisqu'il semble contredire le caractère volontaire de l'action accomplie par colère. Du point de vue éthique, on pourrait comprendre ce déplacement du principe de l'action injuste faite sous l'emprise de la colère au sens métaphorique : on peut dire seulement du point de vue de la délibération que l'archê de l'action est dans celui qui a provoqué la colère chez quelqu'un, lequel a réagi en commettant, lui, une action injuste volontairement mais sans choix délibéré. Mais on peut aussi interpréter ce passage comme un problème juridique et supposer qu' Aristote fait ici allusion à un contexte de droit pénal, et notamment au jugement (cf. krinetai) d'une agression (voire un meurtre), commise sous l'effet de la colère en réaction à une injustice infligée délibérément ${ }^{24}$. Aristote explique en termes éthiques et psychologiques, c'est-à-dire philosophiques, la "déresponsabilisation" légale de la colère provoquée par une injustice.

I.4. Pathos, acte injuste, acte coupable

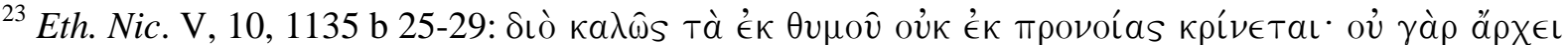

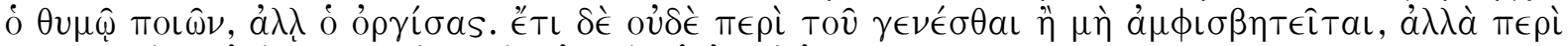

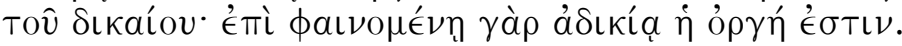

${ }^{24}$ Cf. par ex. T.J. Saunders, Plato's Penal Code, Tradition, Controversy, and Reform in Greek Penology, Oxford, 1991, p. 225, n. 24: "Is a report of jurisprudential opinion, common opinion, or legal practice, and what relevance it has to homicide law in particular".
} 
Je voudrais souligner deux points de cette analyse des passions dans le livre I de la Rhétorique

(a) Le rôle du pathos dans la théorie de l'action. Il faut tenir toutefois compte du fait qu'il s'agit d'une théorie "restreinte" de l'action, puisqu'elle concerne les actes injustes et son contexte est celui de la rhétorique judiciaire et du droit pénal. Le pathos est l'une des causes de l'action, il est identifié au désir irrationnel, dans ses deux formes de désir impulsif (thumos) et désir sensuel (epithumia). On remarquera que dans ce contexte, Aristote tend à identifier la faculté désirante (orektikon) de l'âme avec le pathos passager, par exemple le thumos avec la colère. La réduction du facteur émotif au désir irrationnel permet à Aristote de distinguer la cause motrice (l'impulsion, le désir en acte) et la cause finale (l'objet du désir irrationnel), qui est dans ce contexte spécifique, le plaisir. On verra que dans le livre II, la plupart des passions sont définies comme des états psychiques de souffrance que l'on tend à faire cesser.

(b) Le rôle du pathos dans la juridiction pénale, à savoir dans la définition de l'acte injuste et de la culpabilité. La connaissance du facteur émotif parmi les mobiles de l'acte injuste est indispensable pour bâtir un discours d'accusation ou de défense qui soit efficace, c'est-à-dire persuasif. Comme nous l'avons vu, pour évaluer toute la gravité d'un délit il est fondamental de savoir reconnaître s'il a été commis sous une impulsion irrésistible comme la colère ou par un calcul délibéré.

II. Les passions comme instruments de persuasion (II, 1-11): la "rhétorique des passions"

Venons-en aux passions non plus comme causes de l'action, mais comme causes du changement dans les jugements des hommes. Il s'agit maintenant d'étudier les passions pour les faire naître chez les juges afin d'influencer leur décision. L'appel aux passions devient ainsi un instrument proprement technique de la rhétorique.

\section{II.1. Légitimité de l'appel aux passions}

La première moitié du livre II de la Rhétorique contient une longue analyse des passions (1-11), suivie d'une analyse des caractères (12-17), qui constitue le développement le plus complet et articulé du facteur émotif dans le corpus aristotélicien.

Ici, les passions sont présentées comme des pisteis entechnoi, à savoir des moyens de persuasion "techniques" produits méthodiquement par l'orateur. Ces moyens sont : le caractère (êthos) de celui qui parle, la mise en condition des auditeurs qui consiste justement à leur inspirer une passion et le discours lui-même. Les moyens de persuasion non techniques 
(pisteis atechnoi), sont en revanche des moyens préexistants, indépendants de l'orateur, comme les témoins, les contrats, le texte de la loi (I, 2, 1355 b 35 suiv.). La disposition de l'auditeur est particulièrement utiles dans les procès : "car la manière de voir n'est pas la même selon que l'on aime ou que l'on déteste, que l'on est en colère ou pacifiquement disposé: les choses apparaissent soit radicalement différentes soit d'une importance différente" (II, 1, 1377 b31-1378a1).

De fait, Aristote semble avoir une double position à l'égard de l'appel aux passions dans la Rhétorique. D'une part, en effet, dans les premières pages ${ }^{25}$, il critique résolument l'intérêt exclusif que les technographes de son temps consacraient à l'appel aux passions en l'employant comme un instrument de manipulation et en négligeant le vrai "corps de la persuasion", à savoir la démonstration (l'enthymème). Il considère ici le pathos comme une preuve (pistis) accessoire. Mais ensuite en I, 2 il réintègre l'appel aux passions dans les "preuves techniques" (I, 2, 1356a 1 suiv.) ${ }^{26}$ et il leur consacre l'analyse extrêmement détaillée de la "rhétorique des passions".

De nombreuses interprétations ont été données pour expliquer cette évaluation

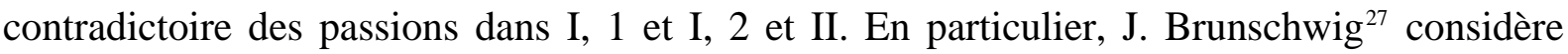
apparente la contradiction: si I, 1 exprime une vision puriste et hautement intellectuelle de la rhétorique, en I, 2 se produit une légitimation du pathos hupo tou logou (1356 a 14): il s'agit de passions produites par le discours et donc non plus en opposition avec l'enthymème. Fortenbaugh ${ }^{28}$ opte, de son côté, pour une "developmental explanation" selon laquelle Aristote

\footnotetext{
${ }^{25}$ Rhet. I, 1, I, 1, 1354 a 11-26: "Mais en fait ceux qui ont composé des traités d'art oratoire n'ont produit, pour ainsi dire qu'une petite partie de cette technique, car les moyens de persuasion constituent le seul élément technique (pisteis entechnon), tout le reste n'est qu'accessoire. Or ils ne disent rien des enthymèmes, ce qui précisément constitue le corps de la persuasion (sôma tês pisteôs), et ils traitent pour l'essentiel de ce qui est extérieur au fait (exô tou pragmatos): car la calomnie, la pitié, la colère et autres semblables passions de l'âme, ne concernent pas le fait mais sont destinées au juré. (...) car il ne faut pas dévoyer le juré en l'amenant à éprouver de la colère, de l'envie ou de la pitié. cela revient à tordre la règle dont on va se servir."
}

${ }^{26}$ Rhet. I, 2, 1356 a 14-24: "Il y a persuasion par les auditeurs quand ces derniers sont amenés, par le discours, à éprouver une passion. Car nous ne rendons pas les jugements de la même façon selon que nous sommes remplis de tristesse ou de gaieté, d'amitié ou de haine. C'est justement à ce seul effet que, d'après nous, tend l'étude des techniciens d'aujourd'hui. Nous éclaircirons cette question point par point quand nous parlerons des passions [...]. (20) Puisque les moyens de persuasion procèdent ainsi, il est évident que leur maîtrise est le fait de quelqu'un qui est capable de manier le syllogisme, de voir clair (theôresai) dans le domaine des caractères et des vertus ou, troisièmement dans le domaine des passions, de voir quelle est chacune des passions, quelle est sa nature, d'où elle naît et comment".

${ }^{27}$ J. Brunschwig, "Aristotle's Rhetoric as a counterpart to Dialectic", dans A. Oksenberg -Rorty (ed.), op. cit. p. 34-55.

${ }^{28}$ W.W. Fortenbaugh, "On the Composition of Aristotle's Rhetoric" $\left(1996{ }^{1}\right)$, dans Aristotle's Practical Side. On his Psychology, Ethics, Politics and Rhetoric, Boston-Leiden, Brill, 2006, p. 389-412. 
serait passé d'une position "puriste", idéaliste et platonicienne, à une conception plus "pratique" de la rhétorique, qui emploie les passions comme "remedial mesures", adaptées à la sensibilité et à la médiocrité des auditeurs ${ }^{29}$.

En réalité, cette contradiction est facile à resoudre sans avoir besoin d'envisager deux phases distinctes dans la pensée d'Aristote. La critique de I, 1 est adressée à un usage exclusif, abusif et incorrect, "sauvage" et déréglé, que faisaient des passions ses contemporains. En revanche, l'admission en I, 2 des passions parmi les pisteis entechnoi, indique un différent usage, correct, issu d'une méthode et subordonné au logos. Nous allons voir, plus loin, un exemple "en acte" de la stratégie du "bon usage des passions" de la part de Lysias.

\section{II.2. La "rhétorique des passions". Quelles passions et quelle méthode?}

Aristote commence par définir les passions en général et les paramètres de son analyse:

Les passions sont les causes par les quelles les hommes, en subissant un changement, diffèrent dans leurs jugements, elles sont suivies de peine ou de plaisir, par exemple la colère, la pitié, la crainte etc. ainsi que leurs contraires. L'examen de chacune d'elles doit être divisé en trois. Je veux dire, par exemple, au sujet de la colère: quelles sont les dispositions des colériques, quelles sont les personnes contre qui on se met d'ordinaire en colère, et à quels sujets. Car si nous ne connaissions qu'un ou deux de ces éléments, mais pas tous, il serait impossible de produire la colère. Il en va de même pour les autres passions. De la même façon que, dans les parties précédentes, nous avons décrit et distingué les prémisses, de la même façon au sujet des passions, nous allons procéder et distinguer selon le même critère ${ }^{30}$.

\footnotetext{
${ }^{29} C$. par ex., Rhet. III, 14, 1415 a 22-35: "La fonction la plus nécessaire de l'exorde, celle qui lui est propre, est donc de faire savoir à quoi tend le discours (...). Tous les autres procédés dont on use, ne sont que des remèdes et sont communs à tous. Les sources dont on les tire sont l'orateur, l'auditeur, l'affaire et l'adversaire. (...). Les procédés visant l'auditeur font appel à divers ressorts: le rendre bienveillant ou le mettre en colère et, quelque fois, le rendre attentif ou l'inverse"; I, 2, 1357 a 11: "...car le juge est a priori un homme simple; II, 21, 1395b 1: "Si les maximes sont d'une grande aide pour les discours, c'est premièrement par ce que les auditeurs sont mal dégrossis".

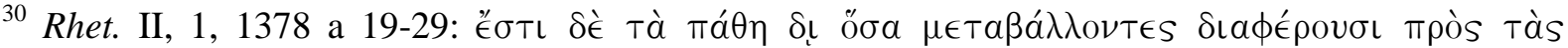

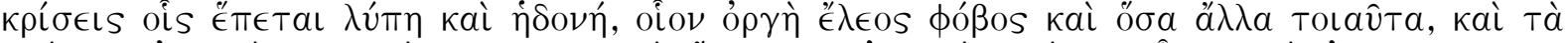

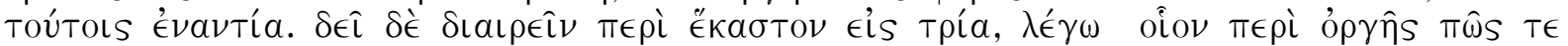

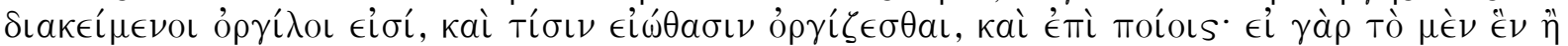

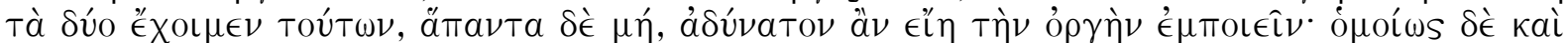

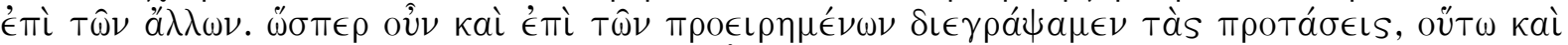

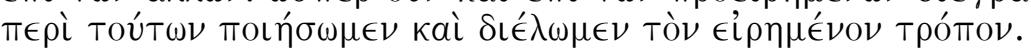


Dans ce passage programmatique qui précède l'analyse détaillée des passions, on peut saisir quelques points essentiels: (a) les passions sont les causes des jugements des hommes. Ou bien, comme le remarque Grimaldi ${ }^{31}$ : les émotions produisent une altération chez les individus qui influence leur faculté critique; (b) elles sont accompagnées de plaisir et de douleur; (c) il faut les analyser selon trois aspects: la disposition de celui qui éprouve une émotion (pôs diakeimenoi), les personnes auxquelles s'adressent les émotions et les choses qui les provoquent; (d) ce critère d'analyse est en vue de leur production (empoiein) chez l'auditeur (les juges).

A propos du point (c), on remarquera une forte ressemblance entre cette méthode tripartite et l'analyse de l'acte injuste de I, 10, 1368 b 3, qui prenait en compte les caractères et les états psychiques de l'agent et du patient. Comme le remarque Grimaldi ${ }^{32}$, ce schéma de diairesis tripartite est en réalité un schéma causal qui sert à rendre compte de la complexité subjective et interactive du phénomène émotif analysé dans la Rhétorique.

Les passions sont ensuite définies et analysées dans les ch. 1-10 par 15 couples de contraires: colère (orgê)/ calme (praotês); amitié (philia)/ haine (misos); peur (phobos)/ assurance (tharrein); honte (aischunê)/ impudence (anaischuntia); obligeance, faveur (charin echein) / désobligeance (acharistein); le trio pitié(eleos) / indignation (nemesis)/ envie (phthonos); émulation (zêlos)/ mépris(kataphronein).

On remarquera tout de suite qu'il ne s'agit pas d'une liste exhaustive d'émotions telles que l'on pourrait les entendre aujourd'hui. Il s'agit surtout d' émotions "sociales" fondamentales: par exemple, un certain nombre d'entre elles sont liées à l'honneur.

Chaque passion est présentée selon un schéma assez constant dans ses points principaux.

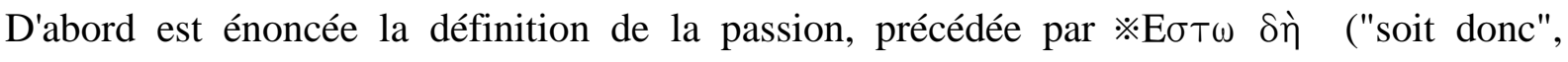
"définissons donc") ${ }^{33}$, la passion est définie comme un "désir" dans le cas de la colère (orexis)

\footnotetext{
${ }^{31}$ Grimaldi, II, p.15.

${ }^{32}$ W.A. Grimaldi, Aristotle, Rhetoric II. A commentary, 1988, p 15-16.

${ }^{33}$ Sur les différents sens de estô dê chez Aristote (hypothetical, resumptive, endoxical) cf.. G. Most, "The use of endoxa: philosophy and rhetoric in Rhetoric", dans D.J. Furley-A. Nehamas (eds), Aristotle's Rhetoric, Philosophical Essays, Princeton, 1994, p. 179-181. Cette formule estô dê, a été considérée par certains (Brandis, Cope-Sandys etc.) comme le signe qu'Aristote se réfère ici à des opinions populaires et non philosophiques, et donc en contraste avec ses théories éthiques. Plus récemment, selon d'autres (Arhnart, Natali, Fortenbaugh, Grimaldi), il se réfère, au contraire, à ses propres doctrines philosophiques. En particulier, selon W. W. Fortenbaugh, Aristotle on Emotion, 1975, p. 16, la formule estô dê signale l'application de la méthode déductive. La formule ei dê...ananchê montre qu'il tire des inférences en rapport logique avec la définition. Most partage la thèse que dans la Rhétorique, Aristote emploie cette formule pour introduire des prémisses afin de démontrer quelque chose. Mais il pense que ces définitions peuvent être indifféremment des théories philosophiques acceptées par Aristote ou des expressions de la vox populi, peu importe qu'elles soient vraies ou fausses: elles doivent seulement servir à construire l'enthymème rhétorique. Je ne partage pas
} 
et de l'amitié (boulesthai), et comme une forme de douleur (lupê) (ou cessation de celle-ci ou insensibilité, dans les cas contraires) dans tous les autres $\operatorname{cas}^{34}$ la cause qui provoque la passion est souvent précédée par le verbe phainomai, ou par phantasia ("imagination") qui indique que la cause est ce qui apparaît comme telle. Prenons l'exemple de la colère:

"Définissons la colère comme le désir, accompagné de douleur, de vengeance manifeste (timôrias phainomenês) ${ }^{35}$, provoquée par ce qui apparaît comme un dédain (dia phainomenên oligôrian) en ce qui regarde notre personne ou celle des nôtres, ce dédain n’étant pas mérité" (Rhet. II, 2, 1378 a 31). La définition est par la suite développée selon les trois paramètres dispositions, personnes, choses. Des distinctions ultérieures concernent les espèces d'une passion (par exemple, pour l'amitié: compagnie, familiarité, parenté) et les incompatibilités entre passions. Par exemple, la peur exclut la compassion à cause de son intensité, la colère et l'assurance excluent la peur car elles ne tiennent pas compte du futur. Il est important de connaître les incompatibilités entre les passions car il ne s'agit pas seulement de les produire, mais aussi d'empêcher qu'elles ne se produisent chez un individu. Les développements sont introduits par la formule "s'il en est ainsi...il s'ensuit nécessairement" (ei dê...ananchê). Par exemple, la définition de la colère se poursuit ainsi: "Si c’est bien en cela que consiste la colère, il s'ensuit nécessairement que l'on se met toujours en colère contre un individu déterminé, par exemple Cléon, et non pas contre l’homme en général "(1378 a 32-34).

Chaque analyse se termine presque toujours par une formule conclusive du genre "Il est clair, il est évident (phaneron de) d'après ce qu'on a dit que..." qui évoque les trois points de l'analyse et le but rhétorique de la passion analysée. Par exemple, nous lisons à la fin de l'analyse de la colère (2, 1380 a): "Quelles sont les personnes contre qui l'on se met en colère, dans quelles dispositions l'on est alors et pour quelles sortes de raisons, tous ces points ont été traités ensemble. Il est clair qu'il nous faudra nous-mêmes, par le discours, mettre le public

la thèse de Most qui me semble trop radicale et se fonder sur une confusion entre la méthode du discours rhétorique décrite dans la Rhétorique et la méthode employée par Aristote lui-même pour exposer la rhétorique comme discipline. Or, il ne me semble pas possible qu'Aristote lui-même puisse accepter d'employer des prémisses qu'il considère fausses dans cette longue et minutieuse analyse des passions de Rhet. II.

${ }^{34}$ La "faveur" (charis) représente une exception. Elle semble avoir un statut particulier car elle signifie l'état émotionnel de celui qui rend un service totalement gratuit. P. Chiron observe (Aristote, Rhétorique, p. 305 n.1) que la charis est l'un des termes grecs les plus difficiles à traduire car elle peut indiquer, selon les contextes, tout aussi bien la bienveillance que la gentillesse ou la reconnaissance.

${ }^{35}$ La première occurrence de phainomenês semble posséder ici la nuance de "manifeste" dans le sens de "public", ce qui apparaît publiquement, et non seulement de "perçu" (aisthêton), comme le comprend D. Konstan (The emotions of ancient Greeks, Toronto, Buffalo, London, 2006, p. 42). C'est la deuxième occurrence qui signifie "perçu", dans le sens de ce qui est "perçu" comme une offense. 
dans une disposition telle qu'il sera porté à la colère, rendre nos adversaires responsables de ce contre quoi l'on se met en colère et pareils à ceux contre qui l'on se met en colère".

L'analyse des passions est complétée par une analyse des caractères (êthê) des auditeurs (ch.. 12-17) que l'orateur doit connaître afin que son discours soit efficace. En particulier, les ch. 12-14 analysent les caractères selon les trois époques de la vie: jeunesse, maturité, vieillesse. Il s'agit d'une description lucide et sans pitié de tous les défauts et de toutes les misères des vieillards. L'un des aspects les plus intéressants de cette analyse est qu'Aristote introduit la composante physiologique du froid et de la chaleur dans le corps humain pour expliquer certaines caractéristiques contraires du caractère des jeunes et des vieux : les jeunes ont, par nature, un tempérament chaud (diathermoi...hupo tês phuseôs, 1389 a 19), cela explique le courage, l' ardeur, l'espoir; les vieux ont un tempérament froid (1389 b 31, katepsugmenoi), ils sont donc lâches, enclins à la peur, et la peur est définie justement comme "une forme de refroidissement" (b 32: ho phobos katapsuxis esti).

Je voudrais maintenant relever ceux qui me semblent les aspects les plus caractéristiques de cette présentation des passions de Rhet. II.

(1) Passions sociales et transitoires. Les émotions sont présentées ici comme les causes du changement des jugements de l'homme. Il s'agit donc de définitions proprement "rhétoriques", à savoir choisies et orientées par un but bien précis, celui de la technê rhétorique: il s'agit de produire ou d'empêcher que la passion ne se produise, de disposer (paraskeuazein) l'âme des auditeurs à éprouver, par exemple, la colère ou la compassion dans le but de la persuasion. Comme nous l'avons déjà observé, cette liste de passions n'est pas une liste complète comme nous pourrions la concevoir de nos jours, mais elle se refère à des passions ayant des caractéristiques conformes au contexte rhétorique dans lequel elles sont présentées.

Il s'agit en premier lieu de passions "sociales" fondamentales, présentées dans leur consistance relationnelle, toujours relatives aux rapports humains et non à un individu isolé. En outre, elles sont présentées comme des dispositions particulièrement transitoires, limitées à des situations particulières, que l'on peut provoquer ou réprimer au moment opportun. Par exemple, la colère est l'émotion la plus mouvementée , mais aussi la plus passagère. C'est aussi le cas de l'amitié, qui est présentée ici comme une forme de désir plus ou moins transitoire, alors que dans Eth. Nic. VIII, 1, 1155a1, l'amitié est définie comme une possession stable: "une vertu ou une forme de vertu". L'aspect stable du facteur émotif se trouve en revanche dans la liste suivante des caractères qui représentent des catégories générales auxquelles il faut rapporter les cas particuliers.

(2) La réduction des émotions au plaisir et à la douleur. Nous avons vu que les passions sont définies dans leur presque totalité comme des formes de douleur (lupê tis) ou de désir (horexis), comme dans le cas de la colère et de l'amitié ( $c f$. boulesthai, le désir rationnel). 
Une exception est constituée par la charis (faveur, obligeance), ce qui semble indiquer plutôt un état d'âme favorable indéfini finalisé à l'utilité, une forme de bienveillance intéressée. Une caractéristique méthodologique de l'analyse des passions du livre II, consiste justement en une tendance à simplifier et réduire la complexité des phénomènes émotifs à un petit nombre d'items de base: plaisir/douleur; diminution/accroissement ou intégration. La colère, par exemple, est un désir douloureux de vengeance pour un manque de considération, le plaisir est dérive du rétablissement d'un équilibre brisé, de la réattestation de soi dans le société après avoir subi une diminution. comme le manque de respect. Nous avons déjà rencontré cette opération de réduction dans Rhet. I, 10, où Aristote, analysant le mécanisme des actions responsables, arrivait à identifier le désir à la cause motrice unifiée et le plaisir à la cause finale unifiée des actes injustes. De plus, nous avons vu de quelle manière en I, 11, le plaisir est défini comme un retour à l'état naturel, comme une reintégration. Leighton ${ }^{36}$, identifie justement dans ce binome plaisir/douleur une partie constitutive des passions qui les distingue des autres phénomènes psychologiques. Il parle à ce propos précisément de pleasure-pain test pour reconnaître une passion dans la Rhétorique.

(3) Hiérarchie, parenté, incompatibilité entre émotions. De fait, la présentation de ces émotions reflète une hiérarchie: en premier lieu, sont prises en considération les émotions sociales fondamentales directes: colère, amitié, peur, honte (et leurs contraires). De fait, la colère est l'émotion par réaction fondamentale, l'exemple paradigmatique même des passions sociales de la rhétorique. Un deuxième groupe est constitué par des émotions "indirectes": pitié, indignation, envie, émulation. Elles se présentent comme une sorte d'émotions au deuxième degré, comme nous le verrons, particulièrement adéquates pour être suscitées ou réprimées dans une assemblée. En outre, comme nous l'avons déjà mentionné en analysant le schéma de présentation de chaque passion, Aristote relève aussi les parentés et les incompatibilités entre passions en vue de ses fins pratiques.

(4) Fonction persuasive et sens moral. Une dernière considération concerne le rapport entre l'usage rhétorique "performatif" des passions et leur sens moral. Il est clair que le but du traité d'Aristote n'est pas d'étudier (theôrein) le sens moral des passions, mais leur fonction persuasive. Toutefois, il n'y a pas d'incompatibilité entre l'étude des passions de la Rhétorique et les analyses de l'Ethique à Nicomaque, il y a plutôt un déplacement du centre d'intérêt ${ }^{37}$.

\footnotetext{
${ }^{36}$ S.R. Leighton, "Aristotle and the Emotions", Phronesis 27 (1982), p. 144-174.

${ }^{37}$ Je ne partage pas sur ce point la position de J. Classen, "Principi e concetti morali nella Retorica di Aristotele", Elenchos, 1989 (1), p. 3-22, qui pense que lorsqu’Aristote emploie dans la Rhétorique des arguments moraux, il ne se réfère pas à ses recherches et à ses réflexions systématiques mais uniquement à la morale populaire, à l'éthique des polloi. Je pense, au contraire, qu' Aristote bâtit son éthique en s'appuyant largement sur la morale populaire et, en particulière, sur l'oratoire. Il n'y a donc pas de contradiction entre les valeurs éthiques de la Rhétorique et les théorisations de l'ouvrage
} 
L'évaluation morale n'est pas le but principal de l'analyse des passions de la Rhétorique, pourtant elle en constitue une composante importante: certains pathê sont propres à un caractère honnête, comme l'indignation, la pitié, l'émulation (qui est définie elle même comme "honnête", epieikês), l'amitié avec les hommes bons, courageux, justes, tempérants. Mais il y a plus: souvent le bien apparaît aussi éminemment persuasif. On touche ici au problème fondamental du contraste entre idéalisme et pragmatisme qui concerne la nature même de la rhétorique: ce qui est persuasif est-il indépendant de la morale ou même immoral? On peut répondre que si le but de la rhétorique n'est pas moral, cela ne signifie pas nécessairement qu'elle soit immorale et qu'elle ne puisse utiliser ce qui est moral. Son but sera plutôt amoral, dans le sens qu'il appartient à un autre registre indépendant: celui du succès et de la persuasion. Si ce qui est persuasif ne doit pas être nécessairement moral, en revanche ce qui est moral est hautement persuasif. Nous trouvons ainsi chez Aristote une sorte de "ruse de la raison" appliquée au bien: le bien a du succès plus souvent que le mal ${ }^{38}$.

Je voudrais maintenant compléter l' analyse des passions dans la Rhétorique d'Aristote par un exemple d'application pratique de l'appel aux passions, de la part d'un orateur célèbre et sans doute connu par Aristote: Lysias.

II.3. Un exemple d'application pratique: Lysias et l'appel aux émotions

Selon Fortenbaugh, Aristote reflète une duplicité d'évaluation concernant l'usage de l'appel aux passions déjà présente dans la rhétorique de son époque et à ce propos il apporte justement l'exemple de deux discours de Lysias où l' on peut saisir cette double attitude en acte $^{39}$.

Le premier est l'un des plus célèbres discours de Lysias, Sur le meurtre d'Eratosthène (I), où Euphilétos oppose à l'accusation de meurtre avec préméditation (phonos ek pronoias) la

d'éthique, qui sont élaborées à partir des premières. La différence est à la limite formelle : Aristote exprime dans la Rhétorique de manière plus simple : metê asapheis, mête akribeis.

${ }^{38}$ Cf. Rhet. I, 1, 1355 a 37: "Mais toujours...le vrai et le meilleur (talêthê kai ta beltiô) se prêtent mieux par nature au syllogisme et, tout simplement, sont plus persuasifs (phusei eusullogistotera kai pithanotera).

${ }^{39}$ W.W. Fortenbaugh, "On the Composition of Aristotle's Rhetoric", art. cit., p. 392: "Apparently the position Aristotle adopts in Rhetoric I,1 is not only ideal and Platonic; it is also a reflection of both actual judicial procedure and the artful pratice of the logographos". Pour les rapports entre Aristote et Lysias, voir, K. J. Dover, Lysias and the Corpus Lysiacum, Berkeley/ Los Angeles, Univ. of California Press, 1968, p. 25 et J.C. Trevett, "Aristotle's Knowledge of Athenian Oratory", Classical Quarterly 46 (1992), p. 371-379. 
légitimité de son acte faisant appel à une loi de Dracon sur l'adultère qui permet au mari outragé de tuer le coupable pris en flagrant délit.

On peut constater dès les premières lignes de l'exorde, qu' Euphilétos vise indéniablement à produire chez les juges les effets émotionnels de la compassion (il n'y a pas le mot mais c'est sous-entendu) à son égard et de l'indignation à l'égard de sa victime:

I, 1: Je donnerai beaucoup, Athéniens, pour que vous me jugiez, dans cette affaire, avec les sentiments que vous auriez pour vous-mêmes à ma place. Je suis sûr que si vous regardiez les maux d'autrui du même oeil que les vôtres, il n'est personne d'entre vous qui ne s'indignât (aganaktoiê) de l'injure qu'on m'a faite ${ }^{40}$.

Mais plus loin, il oppose le texte de la loi aux mensonges et aux artifices de ceux qui cherchent à susciter la colère des juges:

I, 28: Mais vous le savez, n'est-ce pas, juges, les gens qui n'ont pas le bon droit pour eux ne veulent pas reconnaître que leurs ennemis disent la vérité. En revanche, ils recourent, eux, aux mensonges et aux artifices de ce genre pour susciter la colère de l'auditoire contre ceux qui ont pour eux le droit. Lis d'abord la loi.

Par ailleurs, toujours dans l'exorde, Euphilétos exprime son intention de s'en tenir uniquement aux faits:

I, 4-5: Maintenant, juges, j'ai à prouver (epideixai) qu' Eratosthène était bien l'amant de ma femme, qu'il l'avait séduite et qu'il déshonorait mes enfants, qu'il s'est introduit dans ma maison pour m'outrager, qu'il n'existait jusque-là, entre lui et moi, aucune inimitié, que je n'ai pas agi pour l'argent, en vue de m'enrichir, et que je n'avais d'autre intérêt que de me faire justice, conformément aux lois. Je vais donc reprendre depuis ses débuts toute mon affaire; je n'omettrai rien et je dirai la vérité: ma seule chance de salut est de vous dire tout ce qui s'est passé, si je le puis.

L'autre discours est Contre Simon (III), qui concerne un cas de blessure avec préméditation (dikê traumatos ek pronoias) au sens "fort", à savoir avec intention homicide.

\footnotetext{
${ }^{40}$ Fortenbaugh, ibid., p. 402: "That too is Aristotelian. It illustrates in a single passage what Aristotle has in mind when he speaks of a remedial proem derived from an attempt to create goodwill and to arouse anger ".
} 
Journal of Ancient Philosophy Vol. IV 2010 Issue 1

Ici aussi nous trouvons, dans une étroite succession, le propos de rester dans le pragma et l'appel à la compassion.

§46: J'en aurais long encore à vous dire sur son compte, si la loi ne défendait, à votre tribunal, de sortir de l'affaire. Songez-y au moins: ce sont mes adversaires qui ont pénétré de force dans ma maison, eux qui nous ont poursuivis, eux qui nous ont entraînés brutalement hors de notre route.

§48 J'aurais donc droit à votre pitié et à celle de tous mes concitoyens, si j'étais frappé comme le veut Simon. Je le mérite déjà, rien que pour avoir été entraîné, à la suite d'incidents de cette nature, dans de pareils procès.

Les émotions des juges que l'on trouve évoquées chez Lysias ne sont pas nombreuses, elles se réduisent en fait, pour la plupart des cas au couple compassion/indignation : la pitié (eleos) à l'égard des victimes/ la colère et l'indignation (aganaktein, orgê), parfois la haine (misein) à l'égard des oppresseurs, des hubrizontes, des ennemis, parfois la honte (aischunê) à l'égard des victimes de la hubris.

On remarquera que le sentiment fondamental au nom duquel Euphilétos en appelle aux juges est l'indignation pour ce qui lui est arrivé personnellement. Bien que dans le langage oratoire, on considère aganaktein comme un synonyme de orgê et de thumos ${ }^{41}$, cette forme d'indignation/colère assume ici une coloration particulière, par rapport à la définition aristotélicienne de la Rhétorique (II, 2, 1378 a 31) où elle apparaît comme un pathos essentiellement individuel, provoqué par une injustice qui atteint notre identité personnelle ou celle de nos proches, de nos amis ou familiers, considérés comme un prolongement de nous mêmes. L'indignation des juges en revanche, concerne une injustice, qui est arrivée à une autre personne, à un autre citoyen égal à nous, et qui nous concerne dans la mesure où cela aurait pu également nous arriver. La colère des juges doit donc être d'une espèce différente de la colère personnelle, une sorte d' émotion au deuxième degré, indirecte. On pourrait dire que, par rapport à la classification aristotélicienne, elle se rapproche plutôt - sans pourtant s'identifier à aucune d'elles - du trio d'émotions eleos (pitié)/ nemesis ("indignation")/ phthonos (envie) (Rhet. II, 8-10), qui justement concernent les échecs et les succès immérités des autres.

\footnotetext{
${ }^{41}$ Cf. D.S. Allen, The World of Prometeus. The Politics of Punishing in Democratic Athens, Princeton, 2000, p. 52 et note 6, p. 349.
} 
Par ailleurs, la stratégie principale de ce discours consiste à transformer un cas privé d'adultère en un cas public d'offense à la communauté et par conséquent, le meurtre d'Eratosthène n'est pas une vengeance privée (idia timôria), mais un acte accompli dans l'intérêt de toute la cité (huper tês poleôs apasê, I, 47s). Dans ce cas, la colère suscitée chez les juges apparaît comme une sorte d'extension et de métamorphose de la colère individuelle en une "colère civique" dirigée contre l'injustice ${ }^{42 .}$

On peut donc voir ici, dans un plaidoyer exemplaire, concernant un délit qu'on pourrait appeler "d'honneur", que l' appel à la colère des juges apparaît comme une procédure déclarée et parfaitement légitime dans la pratique judiciaire qui opère la légitimation publique d'une passion individuelle ${ }^{43}$ et la transfiguration d'un cas privé en un cas politique, intéressant toute la cité.

Denys d'Halicarnasse souligne cette technique de Lysias (I, 17, 3):

Tantôt il fait l'éloge des juges, tantôt il dit que l'affaire est d'ordre public, concerne tout le monde et fait partie des affaires dont l'auditoire ne doit pas se désintéresser.

On peut considérer cette technique comme une application du bon usage de l'appel aux passions, dans le sens aristotélicien, c'est-à-dire un appel aux passion maîtrisé par le logos. Je n'ai pas trouvé dans la Rhétorique d'Aristote une théorisation exacte de cette pratique. Mais elle semble relever de la technique d' "amplification" (auxesis) pour montrer l'importance de ce dont on parle, qu'Aristote insère parmi les éléments de l'épilogue (III, 19, 1419 b 10 suiv.), et aussi dans l'exorde ( $c f$. dans les III,14, 1415 b1: "Ce qui rend les auditeurs attentifs, ce sont les affaires d'importance, celles qui les concernent personnellement, les choses extraordinaires et celles qui leur procurent du plaisir"). Dans le même esprit apparaît l'emploi des maximes dans les discours : Aristote dit qu'elles sont d'une grande aide dans les discours parce que les auditeurs sont mal dégrossis et "sont contents si quelqu'un parlant en général rencontre les opinions qu'ils ont eux-mêmes dans les cas particuliers "(II, 21, 1395b 1).

Conclusions: la causalité des passions et la nature de la Rhétorique d'Aristote

\footnotetext{
${ }^{42}$ Cf. D. Konstan, The emotions of ancient Greeks, Toronto-Buffalo-London, 2006, p. 68.

${ }^{43}$ D.S. Allen, "Angry bees, wasps, and jurors: the symbolic politics of orgê in Athens", dans S. Braund - G.W. Most, op. cit., p. 76-98, explique l'importance fondamentale de l'appel à la colère des juges par le fait que cette émotion était en réalité une valeur hautement morale, intégrée de manière indissoluble dans les définitions de loi et de justice. Allen synthétise bien cette situation dans The World of Prometeus, p. 62: "The practice of punishing transformed a moment of anger into a moment of justice. The mouvement from the anger to justice or from the personal to the political occurred as a negotiation of honor and of status roles within the community".
} 
Journal of Ancient Philosophy Vol. IV 2010 Issue 1

Revenons maintenant aux deux questions posées au début de cette analyse.

La première concernait les formes de causalité attribuées aux passions par Aristote. Comment s'articulent-elles entre elles? Qu' y a-t-il en commun entre l'évaluation des passions comme mobiles de l'action dans la rhétorique judiciaire de I, 10-15 et l'analyse des passions comme instruments de la persuasion de II, 1-11? Peut-on reconduire leurs actions à un modèle unique de causalité?

La deuxième concernait le rapport entre la théorie des passions et la compréhension de la nature du traité rhétorique lui-même. Le traitement aristotélicien des passions peut-il nous permettre de mieux comprendre la nature du traité rhétorique lui-même?

Commençons par la première.

Tout d'abord, il faut tenir compte du fait que les deux contextes où sont analysées les passions dans la Rhétoriques présentent deux perspectives différentes. Comme le dit bien Roland Barthes, dans le livre I, nous sommes dans la perspective de l'orateur, de "l'émetteur" du message. Celui-ci a besoin de connaître les mobiles de l'action délictueuse pour construire un discours d'accusation ou de défense. Dans le livre II, en revanche, la perspective est celle du public, à savoir du "récepteur" du message, qui doit juger telle action et qu'il faut influencer dans son jugement ${ }^{44}$. Ici les passions sont évaluées comme causes du jugement et comme instruments de persuasion. Ces deux perspectives entraînent les différences suivantes:

(1) En I, sont prises en compte toutes les passions qui concernent les deux formes de désir irrationnels, le thumos et l'epithumia. En II, il s'agit uniquement de passions sociales, à savoir celles qui concernent les rapports entre les hommes de la communauté. On remarquera que dans la "rhétorique des passions" du livre II, les passions dictées par le désir sensuel au sens propre, l'epithumia, n'apparaissent pas. Ou bien, elles apparaissent de manière indirecte pour expliquer certains actes qui provoquent les passions ci-dessus. Par exemple, un acte de hubris, dicté par la jouissance de celui qui le fait, provoque la colère de sa victime. Je crois que l'exclusion des pathê en commun avec les animaux s'explique par leur caractère privé et individualiste, ou du moins "asocial". La faim, par exemple, ou le désir de sexe, est un fait privé qui établit un rapport avec autrui de manière accidentelle. Les passions traitées dans Rhet. II, sont au contraire des passions éminemment sociales qui naissent seulement dans les rapports avec les autres êtres humains. D'où l'espace consacré aux passions "rivales" et

\footnotetext{
${ }^{44} \mathrm{R}$. Barthes, "L'ancienne rhétorique", Communications 16 (1970), p. 179: "Le livre I de la Rhétorique est le livre de l'émetteur du message, le livre de l'orateur: il y est traité principalement de la conception des arguments, pour autant qu'ils dépendent de l'orateur, de son adaptation au public, ceci selon les trois genres reconnus de discours (judiciaire, délibératif, épidictique). Le livre II est le livre du récepteur du message, le livre du public: il y est traité des émotions (des passions), et de nouveau des arguments, mais cette fois-ci pour autant qu'ils sont reçus (et non plus, comme avant, conçus)".
} 
Journal of Ancient Philosophy Vol. IV 2010 Issue 1

"compétitives", comme la colère, l'émulation, l'envie, l'inimitié et, dans un certain sens, l'amitié.

(2) En I, les passions sont prises en considération en tant que causes de l'action et le désir est analysé dans son double aspect, de cause motrice et de cause finale. Dans II, les passions sont étudiées en tant qu'outils de persuasion. Mais alors quel genre de causalité exercent les passions pour faire changer l'opinion des juges?

De fait, elles sont provoquées par le discours, donc d'une manière maîtrisée et filtrée. L'effet causal des passions produites chez l'auditeur serait plutôt d' "influencer" son jugement ${ }^{45}$. Il s'agit donc d'une forme de causalité indirecte et plus faible par rapport à la causalité attribuée au facteur émotionnel dans le livre I. On pourrait rapprocher ce genre de causalité des causes "adjuvantes et prochaines" que Cicéron distingue des "causes complètes et principales" chez les Stoïciens ${ }^{46}$. En effet, ces genres de causes ont la fonction de "coopérer" avec une autre cause ou bien de "prépater" ou d' "intensifier" ${ }^{47}$ les effets de la cause principale, qui serait, dans notre cas, la persuasion par les faits présentés par une argumentation bien agencée (l'enthymème). Le fait de susciter une passion est subordonnée à la force de l'argumentation, du moins selon les règles "officielles" de la rhétorique honnête qu' Aristote énonce dans le livre I (cf. I, 1, 1135 a 5 suiv.).

(3) De plus, si le fait de juger dans un tribunal est bien une certaine action, il y a pourtant un décalage entre les passions en tant que moteurs de l'action et les passions que l'on cherche à susciter chez les juges afin de déterminer leur jugement. Ces dernières appartiennent à un registre différent: "censuré", approuvé, officiel. Ce ne sont pas des émotions directes mais au deuxième degré. Par exemple la colère de la personne offensée n'est pas la même que celle du juge qui s'indigne. La première est individuelle, immédiate et réagit à une offense personnelle, la seconde prend une dimension collective et universelle de colère contre l'injustice et pour la défense du bien de la communauté.

Venons-en à notre deuxième question: l'analyse des passions nous permet-elle donc de mieux comprendre la nature du traité aristotélicien?

Prenons à nouveau la définition de la rhétorique dans I, 2, 1355 b 26: "Nous définissons la rhétorique comme la capacité (dunamis) de reconnaître (theôrein) dans chaque cas ce qui est potentiellement persuasif".

\footnotetext{
${ }^{45}$ Par ailleurs, on remarquera que l'équivalent grec d' " influencer" est "persuader" (agô, peithô). Ce qui montre bien qu'il s'agit d'une action éminemment rhétorique et psychagogique.

${ }^{46}$ Cicéron, Du destin, 39-43 (LS 62 C)

${ }^{47}$ Pour la distinction entre causes complètes, préliminaires, auxiliaires et coopérantes chez les Stoïciens, voir Clément Alexandrin, Mélanges, VIII, 9, 33, 1-9 (SVF II, 351, LS 55 I)
} 
Comme nous l'avons souligné, cette définition montre de manière assez claire que la discipline en question est avant tout théorique, qu'il s'agit, en premier lieu, d' une méthode qui touche à la theoria de la persuasion, à savoir la connaissance des mécanismes qui président à la persuasion, en d'autres termes: la connaissance des causes de la persuasion.

Aristote la compare à d'autres technai, comme la médecine, dont la tâche (ergon) n'est pas de produire la santé mais de bien soigner :

...voilà ce qui est évident, et aussi que sa tâche n'est pas de persuader (ou to peisai ergon autês) mais de reconnaître (idein) ce que chaque cas comporte de persuasif, comme cela se passe aussi dans toutes les autres techniques (car la médecine non plus n'a pour tâche de produire la santé [to hugia poiêsai], mais d'en approcher le plus possible, car il est possible, même lorsque les patients sont incapables de recouvrer la santé, de les bien soigner )(I, 1, 1355b 10-14).

Aristote, souligne un peu plus loin (2, 1356 b 29), qu'aucune technique ne porte sur le particulier:

La médecine, par exemple, n'examine pas ce qui est sain pour Callias, mais bien ce qui l'est pour celui ou ceux qui ont telle constitution: c'est là en effet ce qui relève de la technique, le particulier au contraire est indéfini et ne peut faire l'objet d'une science, la rhétorique non plus n'étudiera pas l'opinion admise (to kath' hekaston endoxon) par Socrate ou Hippias, mais l'opinion admise par telle sorte de gens, comme le fait aussi la dialectique".

Cette remarque attire l'attention sur le fait que le sujet direct auquel s'applique l'art, aussi bien de la persuasion que de la guérison, c'est l'individu particulier, qui est dans son individualité un facteur inconnu. La nature humaine individuelle appartient par excellence au domaine de l'instabilité : la réponse du corps au soin du médecin, de même que la réaction pratique (émotive ou rationnelle) de chaque membre de l'auditoire aux instruments persuasifs de l'orateur ne sont pas garantie. Aussi bien le médecin que l'orateur doivent tenir compte du "facteur humain" individuel et le relier à des principes généraux.

La médecine semble représenter un cas paradigmatique de science pratique où la connaissance des causes est comprise. A ce propos, Aristote esquisse la figure des médecins philosophes qui s'intéressent aussi aux principes naturels et semblent rejoindre les physiciens. En Eth. Nic. X, 10, 1181 b 2, Aristote dit que les médecins ne se forment pas dans les manuels (ek tôn suggrammatôn), mais en distinguant entre non experts (oi anepistêmoi ) et experts 
Journal of Ancient Philosophy Vol. IV 2010 Issue 1

(empeiroi), il précise que les manuels où les auteurs s'efforcent de regrouper les cas individuels en classes de dispositions, sont en revanche utiles pour ces derniers. Dans De sensu, 436 a 16, Aristote parle des médecins "philosophes" qui complètent leur art avec l'étude des principes de la physique:

Il faut étudier ce qu'est <chacune de ces propriétés> et quelles sont les causes qui les provoquent. D'autre part, il revient au naturaliste d'examiner les premiers principes de la santé et de la maladie. En effet, il ne peut y avoir ni santé ni maladie chez les êtres privés de la vie. C'est pourquoi l'on peut dire de la plupart de ceux qui étudient la nature ainsi que des médecins qui s'adonnent à leur art avec le plus de philosophie, que les premiers parachèvent <leur recherche> avec la médecine et que les autres commencent la médecine par la physique.

La même idée est exprimée dans De Vit. 5, 480b 21-30 :

Nous avons donc traité à peu près complètement ce qui concerne la vie et la mort ainsi que les questions liées à cet examen. D'autre part, ce n'est pas seulement au médecin, mais aussi, jusqu'à un certain point au naturaliste, qu'il revient d'exposer les causes de la santé et de la maladie (mechri tou tas aitias eipein). Les aspects, sous lesquels ils diffèrent et sous lesquels diffèrent aussi les objets qu'ils étudient, ne doivent pas nous échapper, bien que les faits montrent que, jusqu'à un certain point, leurs domaines respectifs ont une certaine affinité. En effet, tous les médecins cultivés et curieux (hosoi kompsoi kai periergoi) disent quelque chose sur la nature et jugent convenable d'y puiser leurs principes et, parmi ceux dont l'affaire est d'étudier la nature, les plus habiles terminent en quelque sorte $\mathrm{l}<$ leur recherche $>$ avec les principes de la médecine.

(trad. P.-M. Morel)

On peut donc en conclure qu'Aristote envisage une figure de médecin accompli et philosophe, qui possède aussi une double formation, empirique et théorique, en physique et en physiologie, qui connaît les causes générales et les principes de la santé et de la maladie. Il connaît les causes de la nature, et au moment d'agir, d'opérer la guérison, il prend en considération les cas individuels et la cause proche qu'il relie aux principes généraux.

Dans Metaph. Z, 7, 1032b 1 suiv. Aristote, analyse les productions (poieseis) de l'art, dont la forme est présente dans la pensée du praticien. Il donne l'exemple de la médecine et décrit le raisonnement du médecin qui cherche à produire la guérison en reliant le cas particulier à la forme de la santé qui est présente dans son esprit en tant que science: puisque la santé 
consiste en cela, si l'on veut obtenir la guérison, il faut que cette chose se réalise, par exemple, un certain équilibre des fonctions du corps, ultérieurement, si l'on veut obtenir cet équilibre, il faut une certaine chaleur. Ainsi le médecin continue à raisonner et avance jusqu'à parvenir, en dernier lieu, à ce qui est en son pouvoir de produire. A ce moment donné, le mouvement qui est effectué par le médecin, à savoir le mouvement qui tend à rétablir la santé, s'appelle production. Les médecins représentent des cas de praktikoi qui réunissent dans le même sujet le théoricien et le praticien.

Mutatis mutandis, on pourrait appliquer le même modèle d'excellence professionnelle à l'orateur et dire que l'orateur accompli possède la forme de la persuasion. Dans chaque cas individuel, il procède comme le médecin en reliant le cas particulier à son but général, en produisant l'outil persuasif le plus approprié à ce cas particulier. Par exemple, il fera usage de l'appel à la pitié à l'égard de la personne à défendre dans un cas où il sait que le jury est composé d'individus particulièrement sensibles au malheur qui l'a frappé, en raison de leur âge et de leur états psychologique du moment (dû par exemple au souvenir d'un malheur récent qui les a frappé). La theôria qu'il possède, en l'occurrence l'enseignement de la Rhétorique d'Aristote, ne lui garantit pas le succès (la persuasion) mais lui fournit la dunamis pour obtenir ce succès. La tâche (ergon) de la discipline est celle de connaître et de reconnaître, de theôrein les causes de la persuasion, le but de l'action du médecin et de l'orateur étant de persuader.

A quoi sert donc de connaître (theôrein) les passions dans la Rhétorique? Pour juger l'action d'une part et pour provoquer (ou entraver) les passions dans le but de persuader.

A la lumière de ces considérations, nous pouvons alors en conclure que le status de la Rhétorique est celui d'une réflexion cognitive sur un champs pratique. La théorisation des passions représente un détour cognitif/descriptif avant le retour à la finalité pratique de la persuasion.

Bibliographie

Allen D.S., The World of Prometeus. The Politics of Punishing in Democratic Athens, Princeton, 2000.

Allen D.S., "Angry bees, wasps, and jurors: the symbolic politics of orgê in Athens", dans S. Braund - G.W. Most, Ancient Anger. Perspectives from Homer to Galen, 2004, p. 76-98. 
Journal of Ancient Philosophy Vol. IV 2010 Issue 1

Aubenque P. "Logos et pathos" et "Sur la définition aristotélicienne de la colère", Revue philosophique 147 (1957), p. 300-317.

Aubenque P., "Logos et pathos. Pour une définition dialectique des passions (De anima, I, 1 et Rhétorique, II)", dans G. Romeyer- Dherbey et C. Viano (éd.), Corps et âme. Sur le De anima d'Aristote, Paris, 1996, p. 7-49.

Barthes R., "L'ancienne rhétorique", Communications 16 (1970), p. 172-223.

Brunschwig J., "Aristotle's Rhetoric as a counterpart to Dialectic", dans A. Oksenberg -Rorty (ed.), Essays on Aristotle's Rhetoric, Berkeley/Los Angeles, London, 1996, p. 34-55.

Chiron P., Aristote, Rhétorique, Paris, 2007.

Classen J., "Principi e concetti morali nella Retorica di Aristotele", Elenchos, 1989 (1), p. 322.

Cooper J., “On Aristotelian Theory of Emotions”, dans E. Oksenberg Rorty (ed.), Essays on Aristotle's Rhetoric, Berkeley/Los Angeles, London, 1996, p. 238-57.

Davidson D., "L' action", dans J. Benoist et alii, Quelle philosophie pour le XXIe siècle? Paris, 2001, p. 300-331.

Dover K. J., Lysias and the Corpus Lysiacum, Berkeley/ Los Angeles, 1968.

Fillion-Lahille J., "La colère chez Aristote", Revue des Etudes anciennes 72 (1970), p. 46-79. Fisher N.R.E., Hybris. A Study in the Value of Honor and Shame in ancient Greece, Warminster, 1988.

Fortenbaugh W.W., "Aristotle's Rhetoric on Emotions", Archiv für die Geschichte der Philosophie 52 (1970), p. 40-70.

Fortenbaugh W.W., Aristotle on Emotion, London, 1975.

Fortenbaugh W.W., "On the Composition of Aristotle's Rhetoric" (1996 ${ }^{1}$ ), dans Aristotle's Practical Side. On his Psychology, Ethics, Politics and Rhetoric, Boston-Leiden, Brill, 2006, p. 389-412.

Grimaldi W.M.A., Aristotle, Rhetoric I. A commentary, New York, 1980.

Grimaldi W.M.A, Aristotle, Rhetoric II. A commentary, New York, 1988.

Konstan K., The emotions of ancient Greeks, Toronto, Buffalo, London, 2006.

Laurenti R., Aristotele, I frammenti dei dialoghi, Napoli, 1987, vol. I.

Leighton S.R., "Aristotle and the Emotions", Phronesis 27 (1982), p. 144-174.

Meyer M. Aristote ou la Rhétorique des passions, Paris, 1989.

Meyer M., Le philosophe et les passions. Esquisse d'une histoire de la nature humaine, Paris, 1991.

Moraux P., Les listes anciennes des ouvrages d'Aristote, Louvain, 1951.

Most G., "The use of endoxa: philosophy and rhetoric in Rhetoric" dans D.J. Furley - A. Nehamas (eds), Aristotle's Rhetoric, Philosophical Essays (Proceedings of Twelfth Symposium Aristotelicum), Princeton, University Press, 1994, p. 167-199. 
Journal of Ancient Philosophy Vol. IV 2010 Issue 1

Saunders T.J., Plato's Penal Code, Tradition, Controversy, and Reform in Greek Penology, Oxford, 1991.

Striker G., "Emotions in Context: Aristotle's Treatment of the Passions in the Rhetoric and His Moral Psychology”, dans E. Oksenberg Rorty (ed.), Essays on Aristotle's Rhetoric, Berkeley-Los Ageles-London 1996, p. 286-302.

Trevett J.C., "Aristotle's Knowledge of Athenian Oratory", Classical Quarterly 46 (1992), p. 371-379. 